OPEN ACCESS

Edited by: Yaakov Bentov

McMaster University, Canada

Reviewed by:

Maria Costanza Chiti,

Cliniques Universitaire

Saint-Luc, Belgium

Francesca Gioia Klinger,

University of Rome Tor Vergata, Italy

*Correspondence:

Kazuhiro Kawamura

kazuhironanami@gmail.com

Specialty section: This article was submitted to

Assisted Reproduction,

a section of the journal

Frontiers in Reproductive Health

Received: 02 December 2020 Accepted: 22 January 2021

Published: 02 March 2021

Citation:

Vo KCT and Kawamura K (2021) Ovarian Fragmentation and AKT

Stimulation for Expansion of Fertile

Lifespan.

Front. Reprod. Health 3:636771.

doi: 10.3389/frph.2021.636771

\section{Ovarian Fragmentation and AKT Stimulation for Expansion of Fertile Lifespan}

\author{
Kim Cat Tuyen Vo and Kazuhiro Kawamura* \\ Department of Obstetrics and Gynecology, Advanced Reproduction Research Center, International University of Health and \\ Welfare, Narita, Japan
}

Since the first baby was born after in vitro fertilization, the female infertility treatment has been well-developed, yielding successful outcomes. However, successful pregnancies for patients with premature ovarian insufficiency and diminished ovarian reserve are still difficult and diverse therapies have been suggested to improve the chances to have their genetically linked offspring. Recent studies demonstrated that the activation Akt pathway by using a phosphatase and tensin homolog enzyme inhibitor and a phosphatidylinositol-3 kinase stimulator can activate dormant primordial follicles in both mice and human ovaries. Subsequent researches suggested that the disruption of Hippo signaling pathway by ovarian fragmentation increased the expression of downstream growth factors and secondary follicle growth. Based on the combination of ovarian fragmentation and Akt stimulation, the in vitro activation (IVA) approach has resulted in successful follicle growth and live births in premature ovarian insufficiency patients. The approach with disruption of Hippo signaling only was also shown to be effective for treating poor ovarian responders with diminishing ovarian reserve, including advanced age women and cancer patients undergoing sterilizing treatments. This review aims to summarize the effectiveness of ovarian fragmentation and Akt stimulation on follicle growth and the potential of IVA in extending female fertile lifespan.

Keywords: Akt stimulation, diminished ovarian reserve, in vitro activation, ovarian fragmentation, premature ovarian insufficiency

\section{INTRODUCTION}

On July 25, 1978, the first baby was born after conception by in vitro fertilization (IVF), establishing a new medical approach, giving the chance to achieve parenthood to more than 10 million couples. Over the past decades, there have been remarkable advances in assisted reproductive technologies, resulting in a higher successful live birth rate (1). However, the treatment outcomes for patients with ovarian dysfunction including premature ovarian insufficiency (POI) and poor ovarian response with diminishing ovarian reserve (POR-DOR) have limited success. Oocyte donation or adoption which cannot help them to have their genetic children are often their only options. Recently, there is a significant rise in the mean age of marriages, leading to a higher rate of advanced age women seeking infertility treatment $(2,3)$. Since ovarian dysfunction cannot be treated by conventional gonadotropin stimulation, new therapeutic interventions are needed to stimulate follicle growth. 
Among the pathways modulating early folliculogenesis, the phosphoinositide 3-kinase (PI3K)/Akt signaling pathway plays a crucial role in the activation of primordial follicles (4-6). Subsequent experiments suggested that the Hippo signaling pathway is also important in the development of follicles (7-9). The disruption of the Hippo signaling pathway by ovarian fragmentation was demonstrated to increase the actin polymerization, leading to the nuclear translocation of Yesassociated protein (YAP), the increased downstream cysteinerich 61, connective tissue growth factor, nephroblastoma overexpressed $(\mathrm{CCN})$ growth factors, and baculoviral IAP repeat containing (BIRC) apoptosis inhibitors (10), resulting in follicle growth enhancement (5, 7-9). The combination of ovarian fragmentation and incubation in the presence of Akt-stimulators developed a new therapy named in vitro activation (IVA) for treating POI patients by our group. Following the IVA treatment, healthy live births have been reported $(7,11,12)$. We also developed drug-free IVA, a simplified approach of IVA, to treat patients with POI at early stage and for POR-DOR patients (13-15). In addition, we demonstrated that laparoscopic ovarian incision could activate the follicles in vivo and was a potential therapy for patients with resistant ovary syndrome (ROS) (16).

These results revealed that ovarian fragmentation and Akt stimulation could improve the infertility treatment outcomes for different categories of ovarian dysfunction. This review summarized the knowledge of ovarian fragmentation and Akt stimulation effectiveness on follicle growth and their potential in female fertility expansion.

\section{THE IMPORTANT ROLE OF AKT SIMULATION IN PRIMORDIAL FOLLICLE ACTIVATION}

The mammalian ovary is a complex organ containing follicles as basic functional units (17). To develop preovulatory follicles containing mature oocytes, a number of small primordial follicles are periodically activated from the pool of primordial follicles to undergo folliculogenesis. Although the mechanisms of selection and activation of dormant primordial follicles are yet not fully clarified, recent studies suggested several important intracellular signaling mechanisms to activate dormant primordial follicles (18). Among these pathways, the PI3K/Akt/forkhead box O3 (FOXO3) pathway is the principal one in primordial follicle activation $(8,18,19)$. This pathway is shown to be activated by granulosa cell-produced Kit ligand (KL) (18). Meanwhile, phosphatase and tensin homolog deleted on chromosome 10 (PTEN) and tuberin/tuberous sclerosis complex (TSC1/2) negatively regulate this pathway (19). In addition, anti-Müllerian hormone $(\mathrm{AMH})$ was described to have inhibitory action to the primordial follicles as it inhibits the KL (20-23). However, AMH action of follicle growth varies by species and follicular stages $(24,25)$.

Once Kit ligand binds its cognate tyrosine kinase receptor (c-kit), the phosphorylation of the intracellular region of c-kit enhances PI3K activity capable of transforming phosphatidylinositol-4,5-bisphosphate (PIP2) into phosphatidylinositol-3,4,5-triphosphate (PIP3). Subsequently, PIP3 stimulates phosphatidylinositol-dependent kinase 1 (PDK1), followed by the increased phosphorylation of Akt and nuclear exclusion of the transcriptional factor, FOXO3 $(4,19,26)$. FOXO3 suppresses the activation of primordial follicles $(18,27)$ (Figure 1). Besides, Akt also stimulates cell growth through inactivation of TSC2, which is achieved by phosphorylating TSC2. The mammalian target of rapamycin (mTOR), the downstream of TSC2, regulates the tissue proliferation (18).

Several studies using the mouse model highlighted the role of the PI3K/Akt/FOXO3 pathway in activating the primordial follicles. The incubation of 3-day aged mouse ovaries in vitro with bpV (pic) (a PTEN inhibitor) with/without 740 YP (a PI3K activator) followed by the transplantation of paired ovaries (treated and untreated) under separate sides of the kidney capsule was presented to activate dormant follicles. After incubating for $48 \mathrm{~h}$, more than half of oocytes in primordial follicles exhibited Foxo3 export. There was also an increased staining of antiMullerian hormone (AMH), suggesting the growth of early follicles (28). After the transplantation to hosts and stimulation with gonadotropins, increases in ovarian sizes and the number of antral follicles in the treated group were evident as compared with paired control, resulting in the delivery of healthy progeny (28). Besides, mutant mice with specific deletion of PTEN was demonstrated to increase granulosa cell proliferation, and ovulatory efficacy as well as to decrease follicle atresia (29). Other studies presented that the deletion of PTEN, TSCI/2, or FOXO3 resulted in an extensive and precocious activation of primordial follicles (30-32). In another work, mice's ovaries cultured with a different PTEN inhibitor, bpV (HOpic) alone for $24 \mathrm{~h}$ had a higher number of follicles at preovulatory stage and slightly higher numbers of pups compared to the controls (33). A recent study represented that a long-term bpV (HOpic) treatment alone for 6 days could promote the primordial follicle activation in bovine ovaries without applying the air-liquid interface cell culture, whereas the bpV (HOpic) treatment affected negatively on the DNA structure and its repair competence (34).

In humans, the incubation of the ovarian tissue with a PTEN inhibitor and/or a PI3K activator was reported to activate primordial follicles $(7,28,35)$. After the long-term incubation with $1 \mu \mathrm{M}$ bpV (HOpic) for 6 days without applying the airliquid interface cell culture, the number of growing follicles in the bpV (HOpic) exposed group increased remarkably. However, the survival rate of secondary follicles from the control group was significantly higher compared to one from the bpV (HOpic) exposed group (59 vs. 27\%) (36). Moreover, another work revealed that a concentration of $\mathrm{bpV}$ (pic) as high as $100 \mu \mathrm{M}$ caused an extensive deterioration to follicles (37). It is consistent with a preliminary experiment in the aforementioned study showing that high doses of bpV (HOpic) (10 and $100 \mu \mathrm{M}$ ) were associated with follicular deformity (36). Meanwhile, in a subsequent experiment, incubation with $100 \mu \mathrm{M} \mathrm{bpV}$ (pic) alone for $25 \mathrm{~h}$ was showed to stimulate the follicle growth suggested by a higher percentage of growing follicles in the bpV (pic) treated group. The quantitative TUNEL assay demonstrated that the follicular viability between $\mathrm{bpV}$ (pic) treated and control group was not significantly different (35). As proposed in these studies, 


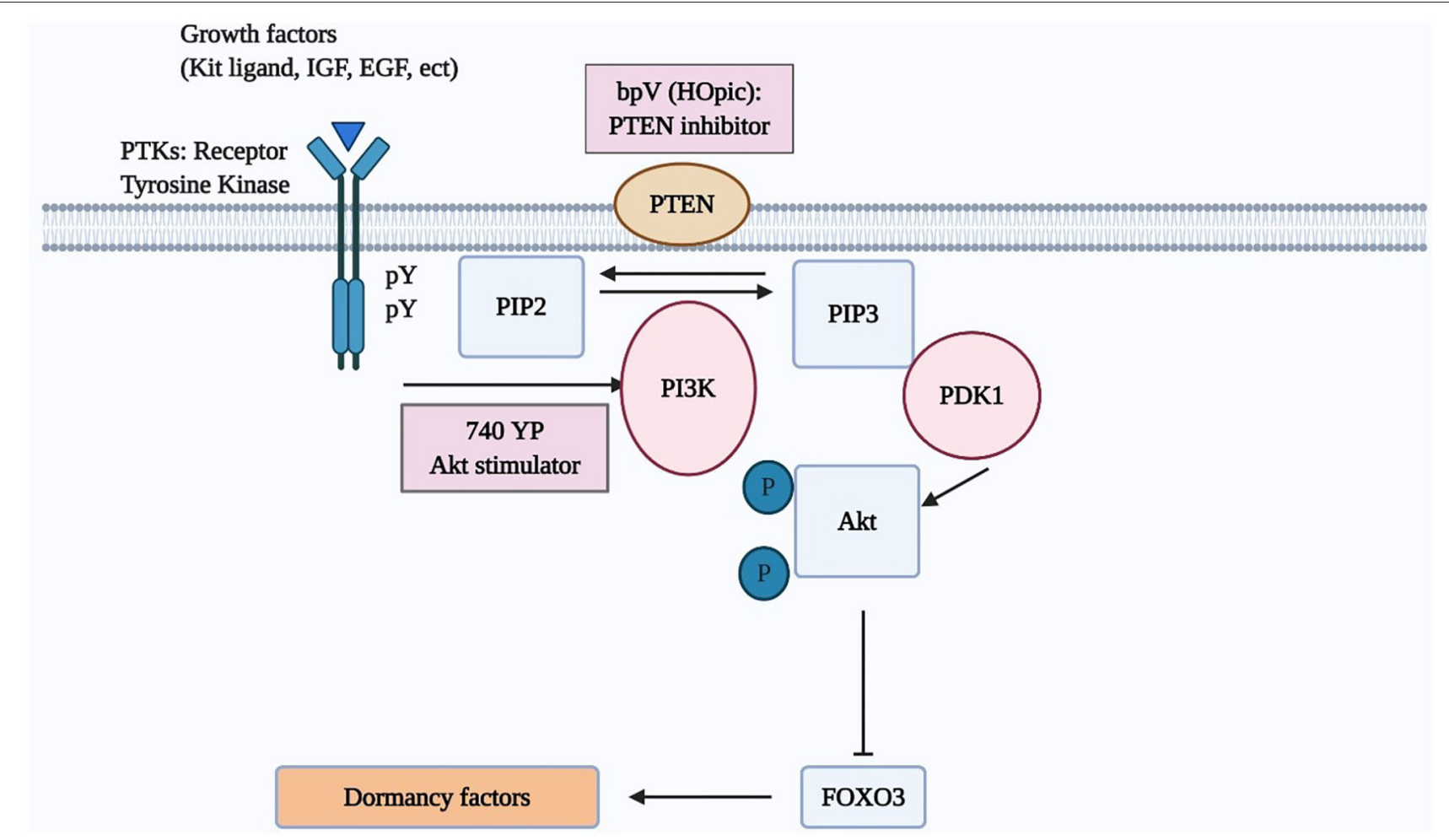

FIGURE 1 | The PI3K/AKT/FOXO3 pathway in oocytes regulates primordial follicle activation. Mouse models were used to investigate the regulation of primordial follicle dormancy. The FOXO3 gene in primordial oocytes serves as a break to prevent the initiation of follicle growth. Activation of upstream RTKs by their cognate ligands (kit ligand, IGF-1, EGF, platelet-derived growth factor [PDGF], VEGF, etc) stimulates the autophosphorylation of intracellular regions of these receptors. Activated receptors then stimulate PI3K activity, leading to increases in PIP3 levels and AKT stimulation. Activated AKT then migrates to the cell nucleus and suppresses FOXO3 actions to promote primordial follicle growth.

the difference in employed PTEN inhibitor, the duration of culture and its procedure may induce different outcomes (35-37).

\section{INDUCTION OF FOLLICLE GROWTH BY FRAGMENTATION OF OVARIAN CORTICAL TISSUES}

The Hippo signaling pathway, initially identified in Drosophila melanogaster, plays a critical role in mechanotransduction and regulates mammalian organ size $(38-40)$. It is modulated by a network of upstream components involved in cell adhesion, shape, and polarity (41). One of these components is actin, a multifunctional protein that forms microfilaments maintaining important cellular processes. The polymerization of globular actin (G-actin) to the filamentous form (F-actin) in the stress fiber has been shown to disrupt the Hippo signaling (42). The Hippo signaling kinase cascade phosphorylates the transcriptional coactivators YAP to promote its cytoplasmic localization and degradation. The disruption of Hippo signaling pathway decreases phosphorylation of YAP, thus increasing nuclear YAP levels (43). Subsequently, increased nuclear YAP interacts with transcription enhancer factor (TEF) to induce transiently the expression of CCN growth factors and BIRC apoptosis inhibitors that have positive effects on cell growth, survival, and proliferation $(10,38,43)$.

In the mammalian ovary, the fragmentation of ovary cortex into small cubes was revealed to disrupt the Hippo signaling pathway by increasing the polymerization of G-actin into F-actin. Consequently, increases in YAP nuclear translocation stimulated the expression of CCN growth factors and BIRC apoptosis inhibitors, resulting eventually in follicle growth $(5,7,44)$ (Figure 2). Furthermore, other studies confirmed that the Hippo signaling pathway works in concert with PI3K/Akt activators to accelerate primordial follicle recruitment $(6,45,46)$.

Using the animal model, several studies demonstrated thoroughly the mechanism of this intracellular signaling pathway. One hour after fragmentation of ovarian cortex from 10-day-aged mice, the ratios of F-actin to G-actin increased transiently and the decrease of pYAP to total YAP ratios were detected, indicating disruption of the Hippo pathway. The CCN2 transcripts in somatic cells were found to increase using realtime RT-PCR. The ability of CCN proteins in the promotion of follicle growth was also demonstrated based on a dose-dependent increase in ovarian explant weights after culturing with CNN2, 3 , 5, or 6 . On the 5th day after grafting under hosts' kidney capsules, a remarkable difference in size was noted between the treated ovaries compared to the paired intact ones. In the 


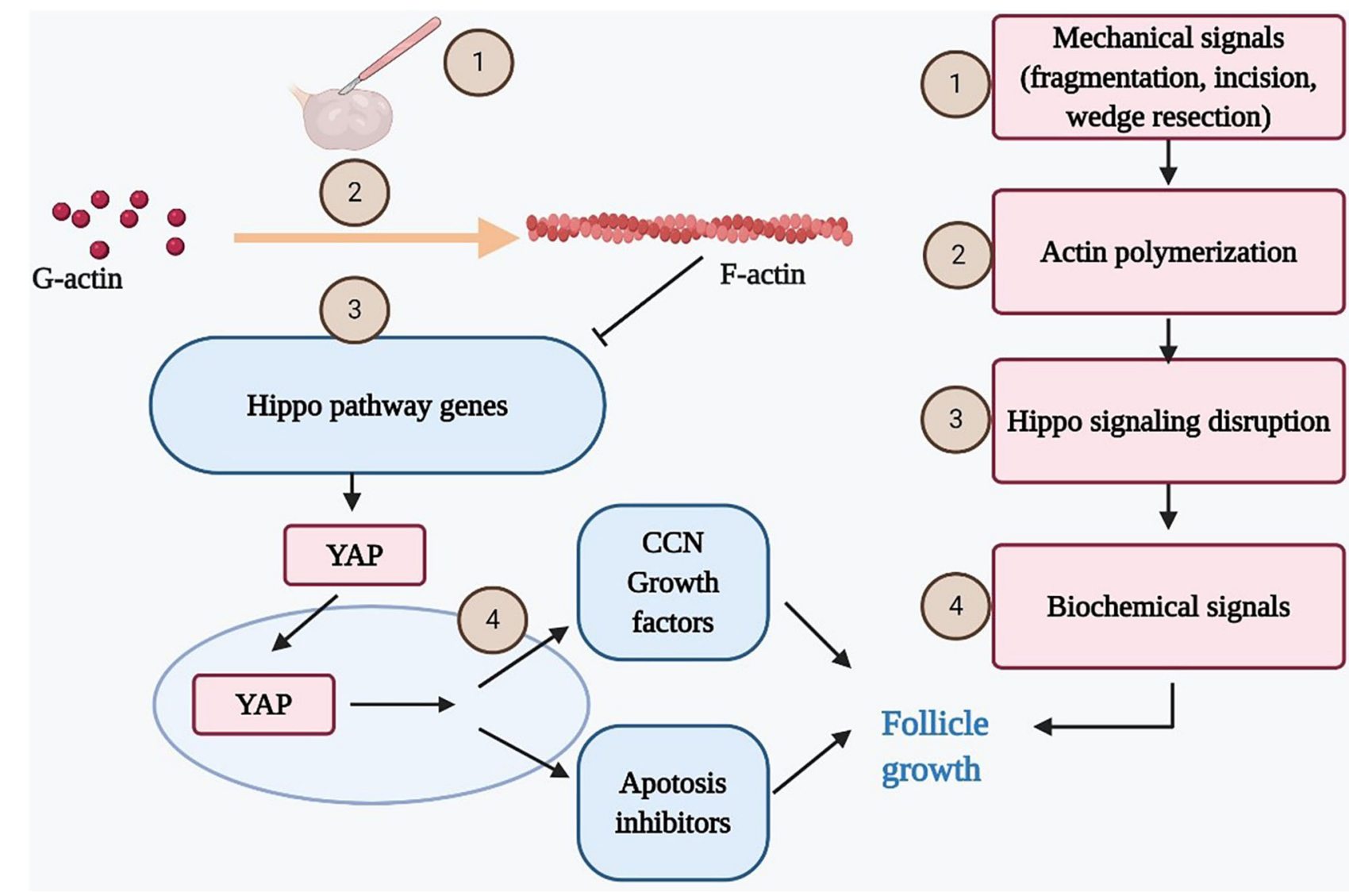

FIGURE 2 | Mechanical force disrupts ovarian Hippo signaling pathway and promotes follicle growth. Mechanical signals incurred by ovarian fragmentation, incision, drilling, or wedge resection lead to actin polymerization that disrupted ovarian Hippo signaling, resulting in nuclear translocation of Yes-associated protein (YAP). Nuclear YAP interacted with transcriptional enhanced associate domain (TEAD) transcriptional factors to increase the expression of downstream biochemical signals (cystein-rich 61, connective tissue growth factor, and nephroblastoma overexpressed [CCN] growth factors and BIRC apoptosis inhibitors), resulting in follicle growth. Ovarian damage-induced follicle growth started with mechanical stimulation but ended with local biochemical changes to promote follicle growth.

histology results, there was an obvious increase in percentages of late secondary and antral/preovulatory follicles, along with the decline in early secondary/primordial follicles. After triggering by an ovulating dose of human chorionic gonadotropin (hCG), fragmented grafts had a 3.1-fold higher number of retrievable oocytes compared to intact grafts (7). The development of early embryos from fertilized mature oocytes retrieved from fragmented grafts was comparable to controls. After ET, healthy pups were delivered (7). An animal study demonstrated that promoting the conversion of G-actin to F-actin by jasplakinolide (JASP) or sphingosine-1-phosphate (S1P) in ovaries of 10-dayold CD-1 mice also increased nuclear YAP and expression of downstream CCN2, leading to the enhancement of follicle growth in vitro and in vivo (44). Besides, the addition of S1P to the culture medium also decreased the follicle atresia and improved the primordial follicle quality (47-49). Similar to the ovarian fragmentation approach, the enzymes degrading the extracellular matrix secreted by granulosa cells were proposed to activate the primordial follicles (50). In contrast, a recent study revealed that S1P treatment could neither activate the primordial follicle nor induce the follicle growth in both mice and human ovaries though the CCN2 gene expression was increased. However, the authors admitted that the longer renewal interval of S1P as compared to one in the study of Cheng et al. (24 vs. $12 \mathrm{~h}$, respectively) could affect the result because the half-life of S1P is as short as $15 \mathrm{~min}$ (51). Genetic studies illustrated the importance of Hippo signaling pathway in regulating folliculogenesis. In mice model, a study indicated that YAP is dispensable for oocyte survival, growth, and maturation (52). In humans, deletion of suppressing actin depolymerization genes as well as other related Hippo pathway genes was identified in subfertile or fertile women (53-57).

\section{THE IMPLEMENTATION OF OVARIAN FRAGMENTATION AND AKT STIMULATION IN INFERTILITY TREATMENT FOR POI PATIENTS}

POI, characterized by early exhaustion of ovarian function, affecting $1-2 \%$ of the population $(58,59)$. Oocyte donation is currently the popular option for infertility treatment in POI 
patients. However, several considerations need to be addressed with oocyte donation. The principal concern is the fact that patients cannot have their genetically related offspring, leading to personal and ethical issues. In some countries, oocyte donation is prohibited due to ethical issues and religious reasons (e.g., many Islamic countries). According to the survey of the International Federation of Infertility Society, 41 out of 215 countries do not allow oocyte donation (60). Moreover, some papers reported that oocyte donation resulted in high-risk pregnancies due to immune compatibility $(61,62)$. A recent meta-analysis concluded that oocyte donation is related to an increased risk of preeclampsia in singleton pregnancies (63).

Given the current knowledge of the PI3K/Akt/FOXO3 and Hippo pathways in follicle growth, IVA has been recently introduced to treat POI women. In this approach, the ovarian cortices are fragmented into small cubes $(1-2 \mathrm{~mm})$ followed by in vitro culture with a PI3K stimulator and a PTEN inhibitor for 2 days and grafting beneath the serosa of the fallopian tubes (Figure 3). Several clinical studies have reported the effectiveness and safety of this treatment. The first pregnancy of this procedure was reported in 27 POI patients with $37.3 \pm 5.8$ years of age, and a long duration of amenorrhea (6.8 \pm 2.1 years). Under laparoscopic surgery, ovaries were removed and cut into strips ( $1 \times 1 \mathrm{~cm}$ with 1-2 mm thickness) before vitrification. Following histological analyses, ovaries from 13 out of 27 patients were found to contain residual follicles. Frozen ovarian strips were thawed and fragmented into $\sim 100$ cubes $(1 \times 1 \times 1 \mathrm{~mm})$, followed by the incubation with Akt stimulating drugs in 2 days. The ovarian cortical cubes were subsequently transplanted beneath the serosa of the fallopian tubes. Following weekly or biweekly transvaginal ultrasound monitoring under ovarian stimulation, follicle growth was found in eight patients. In five patients, mature oocytes were successfully retrieved for intracytoplasmic sperm injection (ICSI) using the husband's sperm. A healthy male baby was delivered at term with normal physical features (7). A subsequent study using the same procedure was conducted on 37 POI patients. 54\% (20/37) of these patients were found to have residual follicles based on histology. Nine out of these 20 women had follicle growth, leading to 24 retrieved oocytes in six patients. After IVF-ET in four patients, three clinical pregnancies were detected, followed by one miscarriage and two healthy live births (11). Another

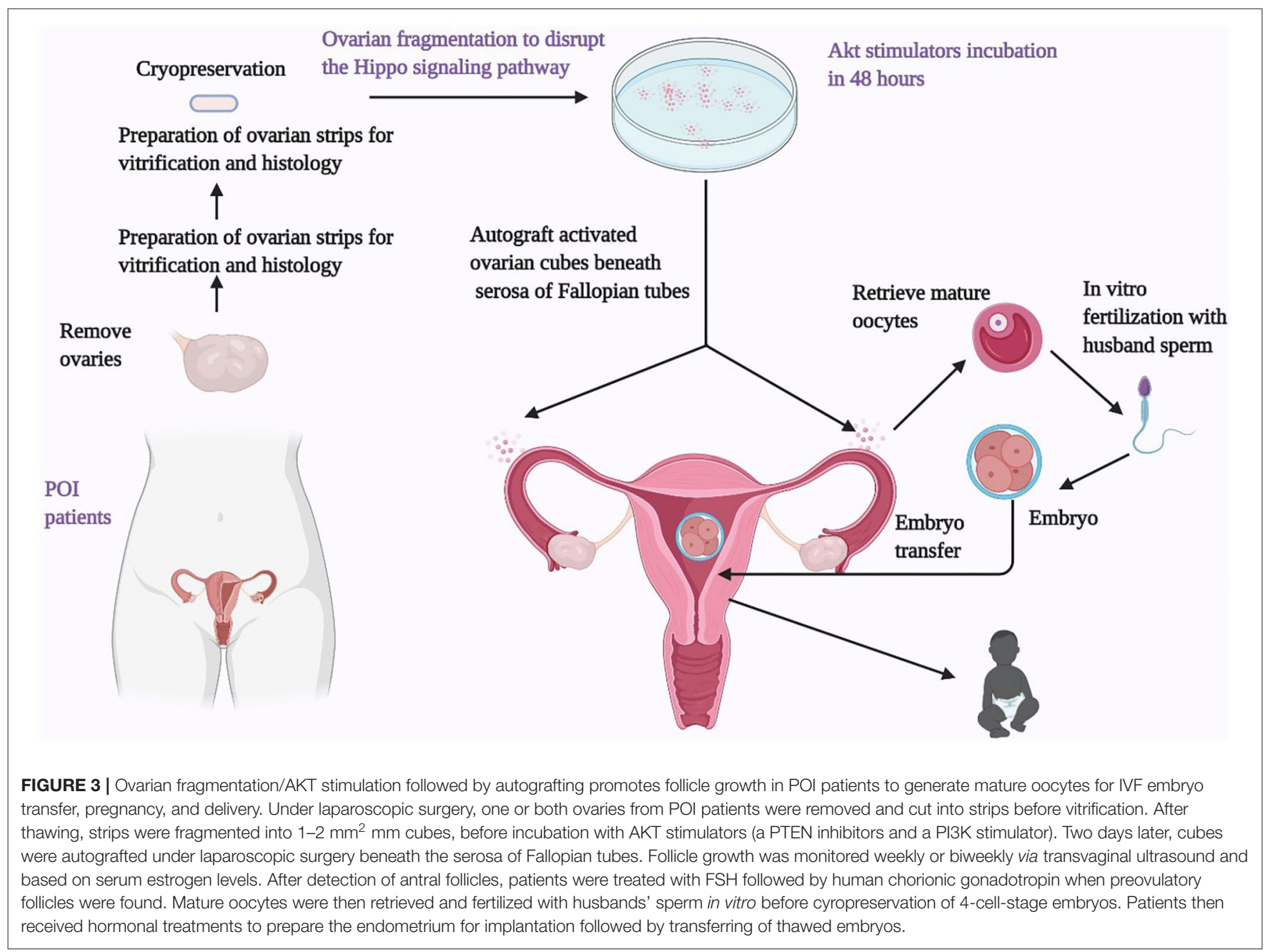


study performed IVA treatment in 14 POI patients with a mean duration since the last menses of 3.8 years. Eleven of the 14 patients had undetectable AMH levels. During the 1-year follow-up period after the IVA procedure, a total of 15 follicle developmental waves were detected in six of the 14 patients (43\%), resulting in six oocytes from four patients. After IVF-ET of retrieved oocytes, one patient delivered a healthy baby boy, with three other frozen embryos (12).

Furthermore, drug-free IVA, the simplified procedure of IVA, was also demonstrated to be successful in treating POI patients at the early stage. In this approach, ovarian cortex is fragmented to disrupt ovarian Hippo signaling followed by the grafting back into remaining ovaries and beneath serosa of Fallopian tubes without tissue culture. Several studies reported successful outcomes by using the drug-free IVA procedure. A case report from Spanish group represented that a 32 -year old patient with elevated FSH levels $(89.9 \mathrm{mIU} / \mathrm{mL})$ and undetectable $\mathrm{AMH}$ levels $(<0.02 \mathrm{ng} / \mathrm{ml})$ became pregnant after the drug-free IVA procedure followed by 20 days of ovarian stimulation (13). Another study from the same group carried out in 14 POI women demonstrated the positive outcome of drug-free IVA. These patients were at the early stage of POI with median age of 33 years (29-36 years), a median length of amenorrhoea of 1.5 years (1-11 years), and a median AMH level of $0.02 \mathrm{ng} / \mathrm{ml}(0.01-0.1 \mathrm{ng} / \mathrm{ml})$. After the drug-free IVA procedure, follicle development was detected in seven patients, and five women achieved successful oocyte retrieval. These five women had six embryo transfers, resulting in four pregnancies (14). Furthermore, a large trial from Chinese group described the promising outcome of the disruption of Hippo signaling pathway by ovarian biopsy and scratching in 80 POI women. Eleven (13.75\%) patients presented with spontaneously ovarian function resumption or follicle growth after human menopausal gonadotropin stimulation. Ten patients underwent oocyte retrieval and IVF, two embryos were transferred to one patient, and one healthy baby was delivered (64).

Although POI patients can achieve pregnancy spontaneously or by other ART therapies (65), these above data demonstrated that IVA yielded better infertility treatment outcomes for POI patients. In a cohort study enrolling 358 young POI patients (mean age: $26.6 \pm 7.9$ years), the spontaneous pregnancy rate was $4.4 \%$ during 13 years (66). In other studies conducted in young POI patients (median age: $30.36-32.5$ years of age), the pregnancy rates ranged from 3.6 to $6.8 \%$ after hormonal therapy with or without ART (67-70). In the second report of IVA study (11), among 37 older POI patients ( $37.6 \pm 4.6$ years of age), twenty patients had residual follicles by the histological analysis, $15.0 \%$ $(3 / 20)$ of patients conceived after the IVA procedure within 1 year and two more patients had cryopreserved embryos pending ET (11). However, these included studies are case series with a limited number of patients, lacking the control group with sham operation. Moreover, long-term neonatal safety is still limitedly examined by genetics examinations. Additional investigations are essential to confirm these findings and ensure the safety of this approach.

A recent study challenged the effectiveness of IVA by xenografting human ovarian cortical tissues to immunodeficient mice. In this study, ovarian tissues from 18 young women were divided into three groups (fresh, slow-frozen, and vitrified). These cortical tissues were fragmented into small cubes followed by with or without culture in Akt stimulators, before xenografting to the peritoneal pockets. The investigators concluded that ovarian fragmentation and Akt stimulation yielded no significant benefits in terms of growing follicle percentages or follicle proliferation rates (71).

However, this study described the follicle density (follicles per $\mathrm{mm}^{2}$ ) which is not highly valid to investigate the benefit of IVA. Since the fundamental mechanism of Hippo pathways and Akt stimulation is improving the development of the primordial follicle with a smaller size to the later stage follicles with a larger size, the follicles density is supposed to not be higher (but even lower) after grafting. Indeed, a significant decrease in follicle density after transplantation was found in this study (71). In terms of the percentage of growing follicles, the duration of 28 days after xenografting is fairly short for evaluating the transition of the primordial follicles to the later stages. Furthermore, the peritoneal pockets as the grafting site may supply a lower blood stream compared to the site under kidney capsule with high vascularization (72). This can have negative impacts on follicle growth.

\section{PERSPECTIVES OF HIPPO SIGNALING PATHWAY AND AKT STIMULATION FOR EXTENDING FERTILE DURATION \\ POR With DOR}

Women have been progressively delaying their childbirth until the third and fourth decades of life, especially in Western countries $(2,3,73)$. This leads to POR with DOR, a natural depletion of oocyte quantity as well as decreases in egg quality, representing unsolved problems in reproductive medicine. The successful outcome of IVA approach in POI patients suggested that this treatment was also beneficial for POR patients with advanced age and severe ovarian dysfunction. Since the activation of primordial follicles could happen spontaneously in DOR condition, the drug-free IVA procedure likely promoted secondary follicle growth. A recent case series study in 11 POR with DOR women at advanced age (30-45) and a median AMH level of $0.04(0-0.8)$ reported that this procedure increased the number of antral follicles following FSH treatment and the number of mature retrieved oocytes per cycle. The fertilization rate and high quality embryo rate were 68.7 and $56.9 \%$, respectively. In consequence, five patients achieved pregnancies, resulting in one live birth, two ongoing pregnancies, and one miscarriage. Moreover, three patients and the miscarriage patient could have cryopreserved embryos (15). Another case series study reported that 13 out of 15 POI and POR with DOR patients who were treated with the drug-free IVA achieved a higher number of antral follicle numbers as well as a higher number of retrieved oocytes as compared to previous IVF outcomes before the IVA treatment. One spontaneous pregnancy and embryo transfer allowed four live births and one ongoing 
pregnancy. Five additional patients and one miscarriage patient have cryopreserved embryos for future transfer (16).

On the other hand, one report recently from Denmark raised questions regarding the effectiveness of IVA. Firstly, they conducted a similar drug-free IVA in 20 POI patients. There were no recorded complications and 12 patients could achieve pregnancies $(74,75)$. There was no significant difference in the number of mature follicles and the AMH levels between the treated group and the control one during 10 weeks of observation (75). Despite high pregnancy rate (60\%) after IVA, an editorial suggested the ovarian fragmentation should be eliminated to treat for DOR patients (76). However, 10 weeks was supposed to be rather short duration for monitoring follicle growth and serum AMH levels (9). In this study, seven out of 20 patients presented an increase in $\mathrm{AMH}$ level and antral follicle counts, indicating the effectiveness of ovarian fragmentation. It is important to note that our earlier publication indicated that serum $\mathrm{AMH}$ levels remained undetectable after IVA treatment when only few follicles reached the preovulatory stage (12).

\section{Fertility Preservation}

As the cancer survival rate among young women has significantly increased recently, efforts to preserve fertility have received significant attention (77). Fertility preservation (FP) by ovarian cryopreservation and autologous transplantation has been practiced in the last two decades and resulted in more than 130 healthy children worldwide $(78,79)$. Because sectioning is essential during the ovarian tissue cryopreservation, in vitro fragmentation and IVA drug treatment can be introduced during the ovarian cryopreservation to enhance the outcome of FP. A study from a Spanish group using 18 human ovarian cortex biopsies from cancer women demonstrated that short term incubation with PTEN inhibitor enhanced the development of growing follicles as well as the surrounding stroma populations without inducing apoptosis. The $\mathrm{AMH}$ concentration in the fresh activated samples was significantly higher compared to the control group (35). In consistence, a recently published study from a Belgium group indicated that fragmentation increased the number of secondary follicles in oncological patients (80).

The ovarian tissue cryopreservation and IVA approach are also likely beneficial to other populations, including women about to be treated with gonadotoxic agents as well as for women with other non-malignant diseases including endometriosis or immune disorders $(79,81)$. Additionally, FP is a favorable option for either unmarried women with severe ovarian dysfunction or women wishing to postpone childbearing for various personal reasons. Of note, some studies reported that the activation of primordial follicle could occur spontaneously after transplantation of frozen-thawed ovarian tissues using the conventional cryopreservation method (82-84) and transient incubation with mTOR inhibitors extended the graft lifespan by preventing the massive activation $(45,85)$. Although the IVA procedure can activate more dormant primordial follicles in frozen-thawed ovarian samples (28), others raised the concern about ovarian endocrine function and reproduction capacity after IVA at long-term goal since the conventional IVA approach provokes an immediate follicular activation (86).

\section{Resistant Ovary Syndrome and Polycystic Ovarian Syndrome}

In addition to the aforementioned perspectives, the application of Hippo signaling pathway can be beneficial to other ovulatory disorders. In detail, some women represent with ovaries unresponsive to endogenous and exogenous gonadotropins, in spite of normal ovarian reserve. This condition has been referred to as ROS, a rare disorder that could not be treated with routine ovarian stimulation (87). Based on the successful outcomes after in vitro fragmentation in POI and DOR patients, it is hypothesized that the incision of ovarian cortex in vivo to disrupt Hippo pathway can stimulate arrested follicles in ROS patients. Interestingly, ovarian incision through laparoscopic surgery was found to promote follicle growth and yield successful oocyte retrieval in seven of 11 ROS patients (16). Although this approach could be a better option compared to IVA, there are no published studies comparing the clinical outcome between IVA and ovarian incision in patients with ovarian dysfunction. Future comparative studies might develop a more efficient and less invasive treatment.

Another ovulatory disorder is polycystic ovarian syndrome (PCOS), the common endocrinopathy affecting approximately $8.7-17 \%$ of women in the reproductive age group (88). Although PCOS patients could achieve pregnancies through current ART practice, some PCOS women faced multiple challenges including poor to an exaggerated response, poor oocyte quality, poor fertilization rate, poor blastocyst conversion, and ovarian hyperstimulation syndrome (88). Ovarian wedge resection and ovarian drilling have been shown to induce follicle growth in PCOS patients, especially in clomiphene citrate-resistant cases $(89,90)$, suggesting induction of follicle growth by alterations in mechanical tensions. There have been several studies that indicated the association between the expression of genes related to Hippo pathway and PCOS condition (91-93). Besides, hypomethylation of the YAP promoter was found to be a key pathogenesis of PCOS (94). Consequently, it is logical to suppose that Hippo signaling pathway is correlated with PCOS. Hippo gene-targeted therapeutics would be effective on fertility and systemic symptoms of PCOS (91). The incision of ovarian cortex or the use of pharmacologic agents targeting this pathway could be applied to PCOS patients to normalize follicle growth and ovulation while minimizing the damage to ovarian reserve. Further studies are necessary to evaluate the safety and efficacy of the ovarian incision in PCOS patients.

\section{CONCLUSION}

In conclusion, social changes and the increasing desire for parenthood of infertile couples have increased the range of ART. The implementation of ovarian fragmentation and Akt stimulators can increase the chance to conceive genetically 
related children for various types of poor prognostic infertile women, leading to the expansion of modern infertility treatment. However, the discussed studies involve a small group of patients, further confirmation by better designed studies is essential for the wide clinical implementation of IVA therapy. The necessity of preparation of a control group with sham operation of IVA makes it difficult and ethically unjustified. Besides, it is necessary to develop a less invasive method to predict the presence of residual follicles before ovariectomy as well as an alternative approach to disrupt Hippo signaling pathway (e.g., actin polymerization-enhancing reagents) to improve the efficiency of IVA.

\section{REFERENCES}

1. Niederberger C, Pellicer A, Cohen J, Gardner DK, Palermo GD, O’Neill CL, et al. Forty years of IVF. Fertil Steril. (2018) 110:185-324.e5. doi: 10.1016/j.fertnstert.2018.06.005

2. Martin JA, Hamilton BE, Osterman MJK, Driscoll AK, Drake P. Births: final data for 2016. Natl Vital Stat Rep. (2018) 67:1-55.

3. Mathews TJ, Hamilton BE. Mean age of mothers is on the rise: United States, 2000-2014. NCHS Data Brief. (2016) 232:1-8.

4. Reddy P, Zheng W, Liu K. Mechanisms maintaining the dormancy and survival of mammalian primordial follicles. Trends Endocrinol Metab. (2010) 21:96-103. doi: 10.1016/j.tem.2009.10.001

5. Hsueh AJ, Kawamura K, Cheng Y, Fauser BC. Intraovarian control of early folliculogenesis. Endocr Rev. (2015) 36:1-24. doi: 10.1210/er.2014-1020

6. Devos M, Grosbois J, Demeestere I. Interaction between PI3K/AKT and Hippo pathways during in vitro follicular activation and response to fragmentation and chemotherapy exposure using a mouse immature ovary model. Biol Reprod. (2020) 102:717-29. doi: 10.1093/biolre/ioz215

7. Kawamura K, Cheng Y, Suzuki N, Deguchi M, Sato Y, Takae S, et al. Hippo signaling disruption and AKT stimulation of ovarian follicles for infertility treatment. Proc Natl Acad Sci U S A. (2013) 110:17474-9. doi: 10.1073/pnas. 1312830110

8. Kawamura K, Kawamura N, Hsueh AJ. Activation of dormant follicles: a new treatment for premature ovarian failure? Curr Opin Obstet Gynecol. (2016) 28:217-22. doi: 10.1097/GCO.0000000000000268

9. Hsueh AJW, Kawamura K. Hippo signaling disruption and ovarian follicle activation in infertile patients. Fertil Steril. (2020) 114:458-64. doi: 10.1016/j.fertnstert.2020.07.031

10. Fu V, Plouffe SW, Guan KL. The Hippo pathway in organ development, homeostasis, and regeneration. Curr Opin Cell Biol. (2017) 49:99-107. doi: 10.1016/j.ceb.2017.12.012

11. Suzuki N, Yoshioka N, Takae S, Sugishita Y, Tamura M, Hashimoto S, et al. Successful fertility preservation following ovarian tissue vitrification in patients with primary ovarian insufficiency. Hum Reprod. (2015) 30:608-15. doi: 10.1093/humrep/deu353

12. Zhai J, Yao G, Dong $\mathrm{F}, \mathrm{Bu} \mathrm{Z}$, Cheng $\mathrm{Y}$, Sato $\mathrm{Y}$, et al. In vitro activation of follicles and fresh tissue auto-transplantation in primary ovarian insufficiency patients. J Clin Endocrinol Metab. (2016) 101:4405-12. doi: 10.1210/jc.2016-1589

13. Fabregues F, Ferreri J, Calafell JM, Moreno V, Borrás A, Manau D, et al. Pregnancy after drug-free in vitro activation of follicles and fresh tissue autotransplantation in primary ovarian insufficiency patient: a case report and literature review. J Ovarian Res. (2018) 11:76. doi: 10.1186/s13048-018-0447-3

14. Ferreri J, Fàbregues F, Calafell JM, Solernou R, Borrás A, Saco A, et al. Drugfree in-vitro activation of follicles and fresh tissue autotransplantation as a therapeutic option in patients with primary ovarian insufficiency. Reprod Biomed Online. (2020) 40:254-60. doi: 10.1016/j.rbmo.2019.11.009

15. Kawamura K, Ishizuka B, Hsueh AJW. Drug-free in-vitro activation of follicles for infertility treatment in poor ovarian response patients with decreased ovarian reserve. Reprod Biomed Online. (2020) 40:245-53. doi: 10.1016/j.rbmo.2019.09.007

\section{AUTHOR CONTRIBUTIONS}

$\mathrm{KK}$ : designed the outline of review. $\mathrm{KV}$ and $\mathrm{KK}$ : wrote the manuscript.

\section{ACKNOWLEDGMENTS}

We thank Dr. Aaron J. W. Hsueh (Stanford University School of Medicine, Stanford, CA) for the critical reading and editing of the manuscript. This work was supported by Japan Agency for Medical Research and Development (AMED) under Grant Number JP20im0210216 (to KK).

16. Tanaka Y, Hsueh AJ, Kawamura K. Surgical approaches of drugfree in vitro activation and laparoscopic ovarian incision to treat patients with ovarian infertility. Fertil Steril. (2020) 114:1355-7. doi: 10.1016/j.fertnstert.2020.07.02:9

17. McGee EA, Hsueh AJ. Initial and cyclic recruitment of ovarian follicles. Endocr Rev. (2000) 21:200-14. doi: 10.1210/edrv.21.2.0394

18. Adhikari D, Liu K. Molecular mechanisms underlying the activation of mammalian primordial follicles. Endocr Rev. (2009) 30:438-64. doi: 10.1210/er.2008-0048

19. Liu K, Rajareddy S, Liu L, Jagarlamudi K, Boman K, Selstam G, et al. Control of mammalian oocyte growth and early follicular development by the oocyte PI3 kinase pathway: new roles for an old timer. Dev Biol. (2006) 299:1-11. doi: 10.1016/j.ydbio.2006.07.038

20. Nilsson E, Rogers N, Skinner MK. Actions of anti-Mullerian hormone on the ovarian transcriptome to inhibit primordial to primary follicle transition. Reproduction. (2007) 134:209-21. doi: 10.1530/REP-07-0119

21. Nilsson EE, Schindler R, Savenkova MI, Skinner MK. Inhibitory actions of Anti-Müllerian Hormone (AMH) on ovarian primordial follicle assembly. PLoS ONE. (2011) 6:e20087. doi: 10.1371/journal.pone.0 020087

22. Campbell BK, Clinton $\mathrm{M}$, Webb $\mathrm{R}$. The role of anti-Müllerian hormone (AMH) during follicle development in a monovulatory species (sheep). Endocrinology. (2012) 153:4533-43. doi: 10.1210/en.20 12-1158

23. Yang MY, Cushman RA, Fortune JE. Anti-Müllerian hormone inhibits activation and growth of bovine ovarian follicles in vitro and is localized to growing follicles. Mol Hum Reprod. (2017) 23:282-91. doi: 10.1093/molehr/gax010

24. Xu J, Bishop CV, Lawson MS, Park BS, Xu F. Anti-Müllerian hormone promotes pre-antral follicle growth, but inhibits antral follicle maturation and dominant follicle selection in primates. Hum Reprod. (2016) 31:1522-30. doi: 10.1093/humrep/dew100

25. Bertoldo MJ, Walters KA, Ledger WL, Gilchrist RB, Mermillod P, Locatelli $\mathrm{Y}$. In-vitro regulation of primordial follicle activation: challenges for fertility preservation strategies. Reprod Biomed Online. (2018) 36:491-9. doi: 10.1016/j.rbmo.2018.01.014

26. Reddy P, Shen L, Ren C, Boman K, Lundin E, Ottander U, et al. Activation of AKT (PKB) and suppression of FKHRL1 in mouse and rat oocytes by stem cell factor during follicular activation and development. Dev Biol. (2005) 281:160-70. doi: 10.1016/j.ydbio.2005.02.013

27. John GB, Gallardo TD, Shirley LJ, Castrillon DH. Foxo3 is a PI3K-dependent molecular switch controlling the initiation of oocyte growth. Dev Biol. (2008) 321:197-204. doi: 10.1016/j.ydbio.2008.06.017

28. Li J, Kawamura K, Cheng Y, Liu S, Klein C, Duan EK, et al. Activation of dormant ovarian follicles to generate mature eggs. Proc Natl Acad Sci U S A. (2010) 107:10280-4. doi: 10.1073/pnas.1001198107

29. Fan HY, Liu Z, Cahill N, Richards JS. Targeted disruption of Pten in ovarian granulosa cells enhances ovulation and extends the life span of luteal cells. $\mathrm{Mol}$ Endocrinol. (2008) 22:2128-40. doi: 10.1210/me.2008-0095

30. Adhikari D, Zheng W, Shen Y, Gorre N, Hämäläinen T, Cooney AJ, et al. Tsc/mTORC1 signaling in oocytes governs the quiescence and 
activation of primordial follicles. Hum Mol Genet. (2010) 19:397-410. doi: $10.1093 / \mathrm{hmg} / \mathrm{ddp} 483$

31. Reddy P, Liu L, Adhikari D, Jagarlamudi K, Rajareddy S, Shen Y, et al. Oocytespecific deletion of Pten causes premature activation of the primordial follicle pool. Science. (2008) 319:611-3. doi: 10.1126/science.1152257

32. Tanaka Y, Park JH, Tanwar PS, Kaneko-Tarui T, Mittal S, Lee HJ, et al. Deletion of tuberous sclerosis 1 in somatic cells of the murine reproductive tract causes female infertility. Endocrinology. (2012) 153:404-16. doi: 10.1210/en.2011-1191

33. Adhikari D, Gorre N, Risal S, Zhao Z, Zhang H, Shen Y, et al. The safe use of a PTEN inhibitor for the activation of dormant mouse primordial follicles and generation of fertilizable eggs. PLoS ONE. (2012) 7:e39034. doi: 10.1371/journal.pone.0039034

34. Maidarti M, Clarkson YL, McLaughlin M, Anderson RA, Telfer EE. Inhibition of PTEN activates bovine non-growing follicles in vitro but increases DNA damage and reduces DNA repair response. Hum Reprod. (2019) 34:297-307. doi: 10.1093/humrep/dey354

35. Novella-Maestre E, Herraiz S, Rodríguez-Iglesias B, Díaz-García C, Pellicer A. Short-term PTEN inhibition improves in vitro activation of primordial follicles, preserves follicular viability, and restores AMH levels in cryopreserved ovarian tissue from cancer patients. PLoS ONE. (2015) 10:e0127786. doi: 10.1371/journal.pone.0127786

36. McLaughlin M, Kinnell HL, Anderson RA, Telfer EE. Inhibition of phosphatase and tensin homologue (PTEN) in human ovary in vitro results in increased activation of primordial follicles but compromises development of growing follicles. Mol Hum Reprod. (2014) 20:736-44. doi: 10.1093/molehr/gau037

37. Lerer-Serfaty G, Samara N, Fisch B, Shachar M, Kossover O, Seliktar $\mathrm{D}$, et al. Attempted application of bioengineered/biosynthetic supporting matrices with phosphatidylinositol-trisphosphate-enhancing substances to organ culture of human primordial follicles. J Assist Reprod Genet. (2013) 30:1279-88. doi: 10.1007/s10815-013-0052-8

38. Pan D. Hippo signaling in organ size control. Genes Dev. (2007) 21:886-97. doi: 10.1101/gad.1536007

39. Hergovich A. Mammalian Hippo signalling: a kinase network regulated by protein-protein interactions. Biochem Soc Trans. (2012) 40:124-8. doi: 10.1042/BST20110619

40. Meng Z, Moroishi T, Guan KL. Mechanisms of Hippo pathway regulation. Genes Dev. (2016) 30:1-17. doi: 10.1101/gad.274027.115

41. Wada K, Itoga K, Okano T, Yonemura S, Sasaki H. Hippo pathway regulation by cell morphology and stress fibers. Development. (2011) 138:3907-14. doi: 10.1242/dev.070987

42. Sansores-Garcia L, Bossuyt W, Wada K, Yonemura S, Tao C, Sasaki H, et al. Modulating F-actin organization induces organ growth by affecting the Hippo pathway. Embo J. (2011) 30:2325-35. doi: 10.1038/emboj. 2011.157

43. Zhao B, Tumaneng K, Guan KL. The Hippo pathway in organ size control, tissue regeneration, and stem cell self-renewal. Nat Cell Biol. (2011) 13:877-83. doi: $10.1038 / \mathrm{ncb} 2303$

44. Cheng Y, Feng Y, Jansson L, Sato Y, Deguchi M, Kawamura K, et al. Actin polymerization-enhancing drugs promote ovarian follicle growth mediated by the Hippo signaling effector YAP. FASEB J. (2015) 29:2423-30. doi: 10.1096/fj.14-267856

45. Grosbois J, Demeestere I. Dynamics of PI3K and Hippo signaling pathways during in vitro human follicle activation. Hum Reprod. (2018) 33:1705-14. doi: 10.1093/humrep/dey250

46. Borreguero-Muñoz N, Fletcher GC, Aguilar-Aragon M, Elbediwy A, VincentMistiaen ZI, Thompson BJ. The Hippo pathway integrates PI3K-AKT signals with mechanical and polarity cues to control tissue growth. PLoS Biol. (2019) 17:e3000509. doi: 10.1371/journal.pbio.3000509

47. Guzel Y, Bildik G, Oktem O. Sphingosine-1-phosphate protects human ovarian follicles from apoptosis in vitro. Eur J Obstet Gynecol Reprod Biol. (2018) 222:19-24. doi: 10.1016/j.ejogrb.2018.01.001

48. Li F, Turan V, Lierman S, Cuvelier C, De Sutter P, Oktay K. Sphingosine-1phosphate prevents chemotherapy-induced human primordial follicle death. Hum Reprod. (2014) 29:107-13. doi: 10.1093/humrep/det391

49. Henry L, Fransolet M, Labied S, Blacher S, Masereel MC, Foidart JM, et al. Supplementation of transport and freezing media with anti-apoptotic drugs improves ovarian cortex survival. J Ovarian Res. (2016) 9:4. doi: 10.1186/s13048-016-0216-0

50. Nagamatsu G, Shimamoto S, Hamazaki N, Nishimura Y, Hayashi K. Mechanical stress accompanied with nuclear rotation is involved in the dormant state of mouse oocytes. Sci Adv. (2019) 5:eaav9960. doi: 10.1126/sciadv.aav9960

51. Pors SE, Harðardóttir L, Olesen H, Riis ML, Jensen LB, Andersen AS, et al. Effect of sphingosine-1-phosphate on activation of dormant follicles in murine and human ovarian tissue. Mol Hum Reprod. (2020) 26:301-11. doi: 10.1093/molehr/gaaa022

52. Lv X, He C, Huang C, Wang H, Hua G, Wang Z, et al. Timely expression and activation of YAP1 in granulosa cells is essential for ovarian follicle development. FASEB J. (2019) 33:10049-64. doi: 10.1096/fj.201900179RR

53. Bione S, Sala C, Manzini C, Arrigo G, Zuffardi O, Banfi S, et al. A human homologue of the Drosophila melanogaster diaphanous gene is disrupted in a patient with premature ovarian failure: evidence for conserved function in oogenesis and implications for human sterility. Am J Hum Genet. (1998) 62:533-41. doi: 10.1086/301761

54. Pisarska MD, Kuo FT, Bentsi-Barnes IK, Khan S, Barlow GM. LATS1 phosphorylates forkhead L2 and regulates its transcriptional activity. Am J Physiol Endocrinol Metab. (2010) 299:E101-9. doi: 10.1152/ajpendo.00534.2009

55. Nagashima T, Kim J, Li Q, Lydon JP, DeMayo FJ, Lyons KM, et al. Connective tissue growth factor is required for normal follicle development and ovulation. Mol Endocrinol. (2011) 25:1740-59. doi: 10.1210/me.2011-1045

56. Pashaiasl M, Ebrahimi M, Ebrahimie E. Identification of the key regulating genes of diminished ovarian reserve (DOR) by network and gene ontology analysis. Mol Biol Rep. (2016) 43:923-37. doi: 10.1007/s11033-016-4025-8

57. Aboura A, Dupas C, Tachdjian G, Portnoï MF, Bourcigaux N, Dewailly $\mathrm{D}$, et al. Array comparative genomic hybridization profiling analysis reveals deoxyribonucleic acid copy number variations associated with premature ovarian failure. J Clin Endocrinol Metab. (2009) 94:4540-6. doi: 10.1210/jc.2009-0186

58. Coulam CB, Adamson SC, Annegers JF. Incidence of premature ovarian failure. Obstet Gynecol. (1986) 67:604-6.

59. Lagergren K, Hammar M, Nedstrand E, Bladh M, Sydsjö G. The prevalence of primary ovarian insufficiency in Sweden; a national register study. $B M C$ Womens Health. (2018) 18:175. doi: 10.1186/s12905-018-0665-2

60. IFFS Surveillance 2016. Global Reproductive Health (2016). 1, 1-143. doi: 10.1097/GRH.0000000000000001

61. van Egmond A, van der Keur C, Swings GM, van Beelen E, van Zijl L, Scherjon SA, et al. Preservation of human placenta facilitates multicenter studies on the local immune response in normal and aberrant pregnancies. $J$ Reprod Immunol. (2013) 98:29-38. doi: 10.1016/j.jri.2013.03.001

62. Stoop D, Baumgarten M, Haentjens P, Polyzos NP, De Vos M, Verheyen G, et al. Obstetric outcome in donor oocyte pregnancies: a matched-pair analysis. Reprod Biol Endocrinol. (2012) 10:42. doi: 10.1186/1477-7827-10-42

63. Schwarze JE, Borda $\mathrm{P}$, Vásquez $\mathrm{P}$, Ortega $\mathrm{C}$, Villa $\mathrm{S}$, Crosby JA, et al. Is the risk of preeclampsia higher in donor oocyte pregnancies? A systematic review and meta-analysis. JBRA Assist Reprod. (2018) 22:15-9. doi: 10.5935/1518-0557.20180001

64. Zhang X, Han T, Yan L, Jiao X, Qin Y, Chen ZJ. Resumption of ovarian function after ovarian biopsy/scratch in patients with premature ovarian insufficiency. Reprod Sci. (2019) 26:207-13. doi: 10.1177/1933719118818906

65. Fraison E, Crawford G, Casper G, Harris V, Ledger W. Pregnancy following diagnosis of premature ovarian insufficiency: a systematic review. Reprod Biomed Online. (2019) 39:467-76. doi: 10.1016/j.rbmo.2019.04.019

66. Bidet M, Bachelot A, Bissauge E, Golmard JL, Gricourt S, Dulon J, et al. Resumption of ovarian function and pregnancies in 358 patients with premature ovarian failure. J Clin Endocrinol Metab. (2011) 96:3864-72. doi: 10.1210/jc.2011-1038

67. Zhang P, Shi Y, Gao X, Wang S, Wang J, Chen ZJ. Clinical analysis of Chinese infertility women with premature ovarian failure. Neuro Endocrinol Lett. (2007) $28: 580-4$.

68. Meden-Vrtovec H, Gersak K, Franić D. Distribution of etiological factors of hypergonadotropic amenorrhea. Clin Exp Obstet Gynecol. (2011) 38:369-72.

69. Chen X, Chen SL, Ye DS, Liu YD, He YX, Tian XL, et al. Retrospective analysis of reproductive outcomes in women with primary ovarian insufficiency 
showing intermittent follicular development. Reprod Biomed Online. (2016) 32:427-33. doi: 10.1016/j.rbmo.2015.12.011

70. Bachelot A, Nicolas C, Bidet M, Dulon J, Leban M, Golmard JL, et al. Long-term outcome of ovarian function in women with intermittent premature ovarian insufficiency. Clin Endocrinol. (2017) 86:223-8. doi: $10.1111 /$ cen. 13105

71. Dolmans MM, Cordier F, Amorim CA, Donnez J, Vander Linden C. In vitro activation prior to transplantation of human ovarian tissue: is it truly effective? Front Endocrinol. (2019) 10:520. doi: 10.3389/fendo.2019.00520

72. Shultz LD, Goodwin N, Ishikawa F, Hosur V, Lyons BL, Greiner DL. Subcapsular transplantation of tissue in the kidney. Cold Spring Harb Protoc. (2014) 2014:737-40. doi: 10.1101/pdb.prot078089

73. Schmidt L, Sobotka T, Bentzen JG, Nyboe Andersen A. Demographic and medical consequences of the postponement of parenthood. Hum Reprod Update. (2012) 18:29-43. doi: 10.1093/humupd/dmr040

74. Lunding SA, Pors SE, Kristensen SG, Andersen CY, Jeppesen JV, Macklon KT, et al. Autotransplantation of fragmented ovarian cortical tissue: a laparoscopic demonstration. Fertil Steril. (2018) 110:1181-3. doi: 10.1016/j.fertnstert.2018.08.024

75. Lunding SA, Pors SE, Kristensen SG, Landersoe SK, Jeppesen JV, Flachs $\mathrm{EM}$, et al. Biopsying, fragmentation, and autotransplantation of fresh ovarian cortical tissue in infertile women with diminished ovarian reserve. Hum Reprod. (2019) 34:1924-36. doi: 10.1093/humrep/dez152

76. Steiner AZ. Evidence that biopsying, fragmentation, and auto-transplantation of ovarian tissue should be abandoned as a treatment of diminished ovarian reserve. Hum Reprod. (2019) 34:1853-4. doi: 10.1093/humrep/dez158

77. Revel A, Revel-Vilk S. Fertility preservation in young cancer patients. J Hum Reprod Sci. (2010) 3:2-7. doi: 10.4103/0974-1208.63113

78. Macklon KT, Jensen AK, Loft A, Ernst E, Andersen CY. Treatment history and outcome of 24 deliveries worldwide after autotransplantation of cryopreserved ovarian tissue, including two new Danish deliveries years after autotransplantation. J Assist Reprod Genet. (2014) 31:1557-64. doi: 10.1007/s10815-014-0331-z

79. Fisch B, Abir R. Female fertility preservation: past, present and future. Reproduction. (2018) 156:F11-27. doi: 10.1530/REP-17-0483

80. De Roo C, Lierman S, Tilleman K, De Sutter P. In-vitro fragmentation of ovarian tissue activates primordial follicles through the Hippo pathway. Hum Reprod Open. (2020) 2020:hoaa048. doi: 10.1093/hropen/hoaa048

81. Hirshfeld-Cytron J, Gracia C, Woodruff TK. Nonmalignant diseases and treatments associated with primary ovarian failure: an expanded role for fertility preservation. J Womens Health. (2011) 20:1467-77. doi: 10.1089/jwh.2010.2625

82. Dolmans MM, Martinez-Madrid B, Gadisseux E, Guiot Y, Yuan WY, Torre A, et al. Short-term transplantation of isolated human ovarian follicles and cortical tissue into nude mice. Reproduction. (2007) 134:253-62. doi: 10.1530/REP-07-0131

83. Smitz J, Dolmans MM, Donnez J, Fortune JE, Hovatta O, Jewgenow K, et al. Current achievements and future research directions in ovarian tissue culture, in vitro follicle development and transplantation: implications for fertility preservation. Hum Reprod Update. (2010) 16:395-414. doi: 10.1093/humupd/dmp056
84. Gavish Z, Peer G, Roness H, Cohen Y, Meirow D. Follicle activation and 'burn-out' contribute to post-transplantation follicle loss in ovarian tissue grafts: the effect of graft thickness. Hum Reprod. (2014) 29:989-96. doi: 10.1093/humrep/deu015

85. Grosbois J, Vermeersch M, Devos M, Clarke HJ, Demeestere I. Ultrastructure and intercellular contact-mediated communication in cultured human early stage follicles exposed to mTORC1 inhibitor. Mol Hum Reprod. (2019) 25:706-16. doi: 10.1093/molehr/gaz053

86. Meirow D, Roness H, Kristensen SG, Andersen CY. Optimizing outcomes from ovarian tissue cryopreservation and transplantation; activation versus preservation. Hum Reprod. (2015) 30:2453-6. doi: 10.1093/humrep/dev210

87. Jones GS, De Moraes-Ruehsen M. A new syndrome of amenorrhae in association with hypergonadotropism and apparently normal ovarian follicular apparatus. Am J Obstet Gynecol. (1969) 104:597-600. doi: $10.1016 / \mathrm{S} 0002-9378(16) 34255-7$

88. Thakre N, Homburg R. A review of IVF in PCOS patients at risk of ovarian hyperstimulation syndrome. Expert Rev Endocrinol Metab. (2019) 14:315-9. doi: 10.1080/17446651.2019.1631797

89. Kandil M, Rezk M, Al-Halaby A, Emarh M, El-Nasr IS. Impact of ultrasoundguided transvaginal ovarian needle drilling versus laparoscopic ovarian drilling on ovarian reserve and pregnancy rate in polycystic ovary syndrome: a randomized clinical trial. J Minim Invasive Gynecol. (2018) 25:1075-9. doi: 10.1016/j.jmig.2018.01.036

90. Abu Hashim H, Foda O, El Rakhawy M. Unilateral or bilateral laparoscopic ovarian drilling in polycystic ovary syndrome: a metaanalysis of randomized trials. Arch Gynecol Obstet. (2018) 297:859-70. doi: 10.1007/s00404-018-4680-1

91. Maas K, Mirabal S, Penzias A, Sweetnam PM, Eggan KC, Sakkas D. Hippo signaling in the ovary and polycystic ovarian syndrome. J Assist Reprod Genet. (2018) 35:1763-71. doi: 10.1007/s10815-018-1235-0

92. Chen ZJ, Zhao H, He L, Shi Y, Qin Y, Li Z, et al. Genome-wide association study identifies susceptibility loci for polycystic ovary syndrome on chromosome 2p16.3, 2p21, and 9q33.3. Nat Genet. (2011) 43:55-9. doi: $10.1038 /$ ng.732

93. Louwers YV, Stolk L, Uitterlinden AG, Laven JS. Cross-ethnic meta-analysis of genetic variants for polycystic ovary syndrome. J Clin Endocrinol Metab. (2013) 98:E2006-12. doi: 10.1210/jc.2013-2495

94. Jiang LL, Xie JK, Cui JQ, Wei D, Yin BL, Zhang YN, et al. Promoter methylation of yes-associated protein (YAP1) gene in polycystic ovary syndrome. Medicine. (2017) 96:e5768. doi: 10.1097/MD.0000000000005768

Conflict of Interest: The authors declare that the research was conducted in the absence of any commercial or financial relationships that could be construed as a potential conflict of interest.

Copyright (๑) 2021 Vo and Kawamura. This is an open-access article distributed under the terms of the Creative Commons Attribution License (CC BY). The use, distribution or reproduction in other forums is permitted, provided the original author(s) and the copyright owner(s) are credited and that the original publication in this journal is cited, in accordance with accepted academic practice. No use, distribution or reproduction is permitted which does not comply with these terms. 